\title{
The evolution of inner disk radius with orbital phase in Circinus X-1
}

\author{
Yanan Wang ${ }^{1,2}$, Guoqiang Ding ${ }^{1}$, and Chunping Huang ${ }^{1,2}$ \\ ${ }^{1}$ Xinjiang Astronomical Observatory, Chinese Academy of Sciences, \\ 150, Science 1-Street, Urumqi, Xinjiang 830011, China \\ email: wangyanan@xao.ac.cn, dinggq@gmail.com \\ ${ }^{2}$ University of Chinese Academy of Sciences, \\ China
}

\begin{abstract}
Using $R X T E$ observations, we investigate the evolution of inner disk radius $\left(R_{\mathrm{in}}\right)$ of Cir X-1 during two cycles and find obvious orbital modulation. We argue that the modulation is attributed to its high orbital eccentricity. The disk luminosity is inversely with the inner disk temperature $\left(k T_{\mathrm{in}}\right)$, which is ascribed to the slow increase of $k T_{\text {in }}$ and, however, the rapid decrease of $R_{\text {in }}$ during the passage for the neutron star to depart from the companion star.
\end{abstract}

Keywords. Compact object, Neutron star, Accretion disk, Circinus X-1

\section{Introduction}

Circinus X-1 (Cir X-1), with an orbital period of 16.6 days (Kaluzienski et al. 1976) and a distance of $5.5 \mathrm{kpc}$ (Case \& Bhattacharya 1998), is a X-ray binary. Its compact star has been considered as a neutron star (NS) since the discovery of type-I X-ray bursts in this source (Tennant et al. 1986). One of the special parameters of Cir X-1 is its high orbital eccentricity ( 0.7-0.9) (Johnston et al. 1999), which makes it a peculiar source. Shirey et al. (1996) found the orbital modulation for the spectrum and quasi-periodic oscillation (QPO) of Cir X-1 and Ding et al. (2006b) found that its power-law (PL) hard tail, evolving on its hardness-intensity diagram (HID) (Ding et al. 2003), is modulated by orbital phase too. At the periastron, a long-term dip was present (Ding et al. 2006a). For explaining the behaviors of observed optical and infrared (IR) emission lines, Johnston et al. (1999) proposed that the accretion and accretion disk of this NS X-ray binary (NSXB) could evolve with orbital phase.

\section{Data Analysis}

With software HEASOFT 6.11 and FTOOLS V.6.11, we choose the observations during two orbital periods (1996 September 21-October 7, 1996 March 8-19) of Cir X-1 to perform our analysis. Following Stewart et al. (1991), the time of zero phase is given by the ephemeris equation

$$
J D_{0}=2443076.87+(16.5768-0.0000353 N) N .
$$

We produce the background-subtracted PCA spectra at different phases. Shirey et al. (1999) used several spectral models to fit the PCA spectra of Cir X-1 and found that the best-fit model is the so-called Eastern model, consisting of a blackbody (BB) and a multicolor disk blackbody (MCD), which are interpreted as the emission from the NS surface and the optically thick accretion disk, respectively. We adopt this model, use it to fit the spectra of Cir X-1 during the two orbital periods, and then get the inner disk 

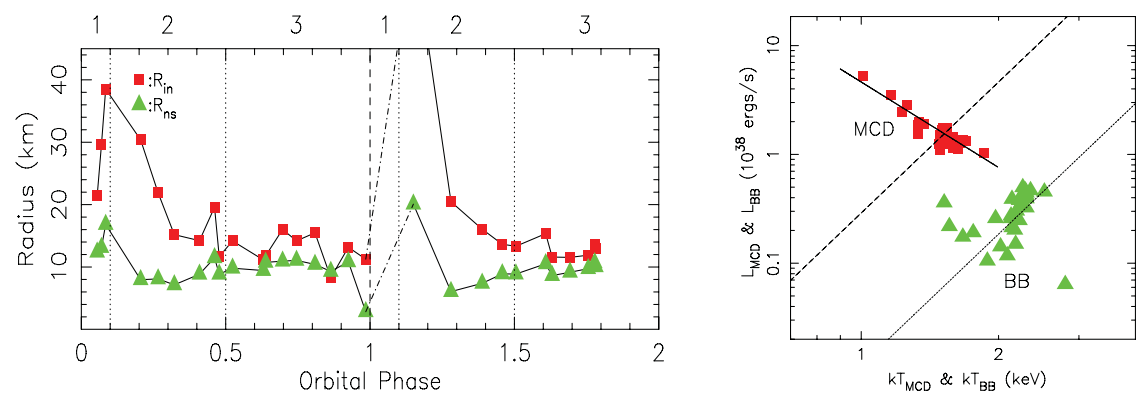

Figure 1. Left panel: the evolution of inner disk radius and the inferred NS radius along orbital phase. Right panel: the luminosities of spectral components (MCD/BB) vs. their characteristic temperatures; the dashed line and dotted line correspond to $L=4 \pi R^{2} \sigma T^{4}$, with $R=15 \mathrm{~km}$ and $R=3 \mathrm{~km}$, respectively; the solid line corresponds to $L \propto T^{-2.6}$.

temperature $\left(T_{\mathrm{in}}\right)$, inner disk radius $\left(R_{\mathrm{in}}\right)$, BB temperature $\left(T_{\mathrm{bb}}\right)$, and BB radius $\left(R_{\mathrm{bb}}\right)$. The BB radii multiplied by a coefficient are considered as the typical NS radius.

\section{Result and Discussion}

As shown in the right panel of Figure 1, the disk emission deviates from the relation of $L \propto T^{4}$, which indicates that $R_{\text {in }}$ is varied, because of $L_{\text {disk }}=4 \pi R_{\text {in }}^{2} \sigma T_{\text {in }}^{4}$. In panel A of Figure 1, one can see that at the periastron (phase 0-0.1) $R_{\text {in }}$ increases abruptly, then from phase 0.1 to the apastron (phase 0.5) $R_{\text {in }}$ decreases rapidly, and, finally, from the apastron to phase 1 the $R_{\text {in }}$ roughly steadies. As suggested by Johnston et al. (1999), at the periastron the large tidal force could make the disk unstable, resulting in large variation of $R_{\text {in }}$; after the apastron until the apastron the disk is formed gradually and meanwhile the NS departs from the companion star, but the disk moves towards the NS due to decrease of radiation pressure; after the apastron, steady accretion takes place on the formed disk. It is obvious that during the passage for the NS to leave the companion star the slow increase of $\left(T_{\text {in }}\right)$ and, however, the rapid decrease of $R_{\text {in }}$ contribute to the inverse correlation between the disk luminosity and the inner disk temperature.

\section{Acknowledgements}

This work is supported by the National Basic Research Program of China (973 Program 2009CB824800) and the Natural Science Foundation of China under grant no. 11143013.

\section{References}

Case, G. L. \& Bhattacharya, D. 1998, ApJ, 504, 761

Ding, G. Q., Qu, J. L., \& Li, T. P. 2003, ApJ, 596, L219

Ding, G. Q., Qu, J. L., \& Li, T. P. 2006a, AJ, 131, 1693

Ding, G. Q., Zhang, S. N., Li, T. P., \& Qu, J. L. 2006b, ApJ, 645, 576

Kaluzienski, L. J., Holt, S. S., Boldt, E. A., \& Serlemitsos, P. J. 1976, ApJ, 208, L71

Johnston, H. M., Fender, R., \& Wu, K. 1999, MNRAS, 308, 415

Shirey, R. E., Bradt, H. V., \& Levine, A. M. 1999, ApJ, 517, 472

Shirey, R. E., Bradt, H. V., Levine, A. M., \& Morgan, E. H. 1996, ApJ, 469, L21

Stewart, R. T., Nelson, G. J., Penninx, W., Kitamoto, S., Miyamoto, S., \& Nicolson, G. D. 1991, MNRAS, 253, 212

Tennant, A. F., Fabian, A. C., \& Shafer, R. A. 1986, MNRAS, 221, 27P 